\title{
CuFusion2: Accurate and Denoised Volumetric 3D Object Reconstruction Using Depth Cam-
} \section{eras}

\author{
Chen Zhang \\ College of Computer Science and Technology, Zhejiang University, Hangzhou 310027, China; \\ Corresponding author: Chen Zhang (e-mail: zhangxaochen@163.com).
}

ABSTRACT 3D object reconstruction from depth image streams using Kinect-style depth cameras have been extensively studied. We propose an approach for accurate camera tracking and volumetric dense surface reconstruction, assuming a known cuboid reference object is present in the scene. Our contribution is threefold: (a) we keep drift-free camera pose tracking by incorporating the 3D geometric constraints of the cuboid reference object into the image registration process; (b) on the problem of depth stream fusion, we reformulate it as a binary classification problem, enabling high fidelity of surface reconstruction, especially in concave zones of the objects; (c) we further present a surface denoising strategy, facilitating the generation of noisefree triangle mesh, making the models more suitable for 3D printing and other applications. We extend our public dataset CU3D with several fresh image sequences, test our algorithm on these sequences and compare them with other state-of-the-art algorithms. Both our dataset and algorithm are available as open-source at https://github.com/zhangxaochen/CuFusion, for other researchers to reproduce and verify our results.

INDEX TERMS 3D object reconstruction, depth cameras, Kinect sensors; open source, signal denoising, SLAM

\section{INTRODUCTION}

Reconstructing the 3D surface model from a sequence of provided range images has been an active research topic during the last decade. In recent years, the emergence of depth cameras based either on structured light (e.g. Asus Xtion, Kinect 1.0) or time-of-flight (ToF) (e.g. Kinect 2.0) sensing offers dense depth measurements directly at a high frame rate as video streams. The KinectFusion algorithm [1] introduced by Newcombe et al. is one of the most seminal works for realtime camera tracking and dense environment reconstruction, turning the depth sensors into consumer-grade 3D scanners. It uses fast iterative closest point (ICP) [2], [3] for camera pose estimation, and a volume known as the truncated signed distance function (TSDF) for scene representation. The connected mesh surfaces are latterly extracted using the marching cubes algorithm [4].

On reconstruction accuracy, however, the KinectFusion algorithm suffers from a number of limitations, including the ICP image registration algorithm that prone to accumulate drift in the presence structure-less surfaces, the inability to recover from drift, and the problem of surface deformation for highly curved and concave zones of the scanned objects [5].

Many researchers have been working on solving these problems. ICP variants such as point-to-plane ICP [6] and generalized ICP (GICP) [7] are proposed for better image alignment. Loop closures are detected and pose graphs are built and optimized online [8]-[12] or offline [13], [14] to produce robust and globally consistent maps. To address the surface deformation problem, Whelan et al. propose the ElasticFusion framework [10], [15] to activate non-rigid model-to-model refinement which also relies on local loop closure detection. Slavcheva et al. propose the SDF-2-SDF algorithm [16], [17] which focus on the small-scale object reconstruction. Similarly, we have also proposed a CuFusion framework [18] for accurate camera localization and object modelling, under the assumption that a known cuboid reference object present in the scene. A prediction-corrected TSDF fusion strategy is applied instead of simple moving average fusion, to resolve the surface deformation problem.

However, the reconstruction quality of [18] relies heavily on the quality of the raw depth measurements. To keep reconstruction fidelity in particularly highly curved zones of the objects, the input depth images should contain as less motion blur as possible, which requires the camera orbiting steadily. Even mild blur in depth measurement caused by slightly faster camera motion may lead to reconstruction failure on sharp edges of the objects. To address these problems, in this paper we introduce a novel approach which is an extension of our previous work [18]. The major contributions are as follows:

- we propose an ICP variant which takes the constraints of the known reference object into account for robust and 
accurate camera pose estimation. With the supplementary information of the reference object, we keep nearoptimal camera tracking for each frame, making it possible for accurate object reconstruction. We build pose graphs and solve for optimized camera poses, and compare them with those without graph optimization, demonstrating that our method is accurate enough;

- we reformulate the data fusion task as a per voxel binary classification task, to keep reconstruction fidelity and resistant to motion blur result from camera jitters;

- we present a denoising strategy, which performs noise reduction directly on the generated volume during scanning, resulting in cleaner mesh surface outputs, taking it one step further for the industrial applicability of the output surface models such as in 3D printing.

We focus on the geometric fidelity, taking depth streams only as input. We perform a qualitative and quantitative evaluation on reconstructions from both synthetic and realworld sequences of the CU3D dataset. Both the camera trajectory and reconstruction accuracy are compared with the state-of-the-art approaches. We show the fidelity of the reconstruction of our method and release our code and dataset to the community for future work.

\section{RELATED WORK}

Simultaneous localization and mapping (SLAM) has been extensively studied. Monocular RGB camera tracking systems such as MonoSLAM [19] and PTAM [20] allow users to get the camera trajectories and sparse point cloud models. Dense reconstruction systems [21]-[23] have also been proposed to replace point cloud based systems. With the advent of Kinectstyle active depth sensors, the KinectFusion [1] algorithm permits dense volumetric reconstruction of the scene in realtime, enabling mesh models output for physics-based augmented reality (AR) [24] and 3D printing [25]. Improved frameworks have then been proposed in the aspects of memory efficiency [26]-[28], large space representation [8], [11], [27], [29], camera trajectory accuracy with loop closure detection and optimization [12], [27], [30], and scene representation such as surfels [31] or hybrid data structure [32].

Some researchers use the structural priors for accurate camera localization. Zhou et al. [33] introduce an approach for robust contour cues extraction and integrate the contour constraints into the registration objective, stabilizing camera tracking in challenging scenarios. High-level features such as planes [34]-[38] and objects [39], [40] are also used as primitives to provide more constraints to camera pose estimation. In this paper, we make full use of the information of a precisely man-made cuboid reference object, namely, the orthogonal and parallel planar facets, the contour cues of certainly known length as constraints for camera localization stabilization. The accurate and robust camera trajectories are latterly used in the model generation process, to integrate single images into consistent models in the global coordinate.

Different dense scene representations have also been explored in the literature. Occupancy mapping using a grid of cells to represent the space has been popular in robotics. A probability of occupancy in each cell is accumulated via Bayesian updates every time a new informative observation is provided [41]. Similarly, the signed distance function (SDF) volumetric representation introduced in [42] is often used in graphics to fuse partial depth scans into one global model. The SDF represents the surface interfaces implicitly as zeros, and the mesh models can be extracted using the marching cubes type algorithm [4]. Instead of volumes, surfels [10], [31], [43], [44] are also exploited to represent the scene. It renders the scene with the surface-splatting technique [45] and reduces the computational complexity and memory overhead compared with the volumetric approaches. The volumetric representation has been reported as difficult to resolve the highly curved and concave details such as the folds in the garment [5] or thin geometries [18] although the voxels are small enough. [18] introduced a prediction-corrected data fusion strategy for geometry details preservation. By storing surface normal and view ray vectors per voxel as additional information, it enables fast correction of the surface where deformation previously accumulated. The main issue of [18] resides in the resistless of motion blur in raw depth measurements, as well as the high memory consumption. In this paper, we introduce a novel method for data fusion, converting the typical moving average to a scheme of per voxel probabilistic binary classification, and resulting in high fidelity of reconstruction in especially sharp geometries.

There have been many popular RGB-D datasets created for the evaluation of indoor 3D reconstruction. The TUM RGB$D$ dataset [46] offers a set of RGB-D images with accurate and time-synchronized ground-truth (GT) camera poses from a motion capture system. It mainly aims at trajectory estimation and lacked the GT scene models. To assess the scene reconstruction, the ICL-NUIM dataset [47] generates both GT poses and models for quantitatively evaluation of the final reconstruction with the synthetic model of one scene. Slavcheva et al. provide the first object dataset with GT CAD models and camera trajectories. It 3D-printed a selection of small objects and scanned them with a markerboard placed below them.

Similarly, we provide a dataset CU3D in [18], with both synthetic and real-world sequences. Our synthetic sequences provide both GT object models and camera poses, whereas the real-world data are generated by scanning six 3D-printed objects and have GT models only. In this work, we extend this dataset with several supplementary sequences. Although we have no per vertex GT for these newly added scans, we verify the reconstruction accuracy by evaluating the total length of the reconstructed model, compared with the Vernier caliper measurements as GT. 


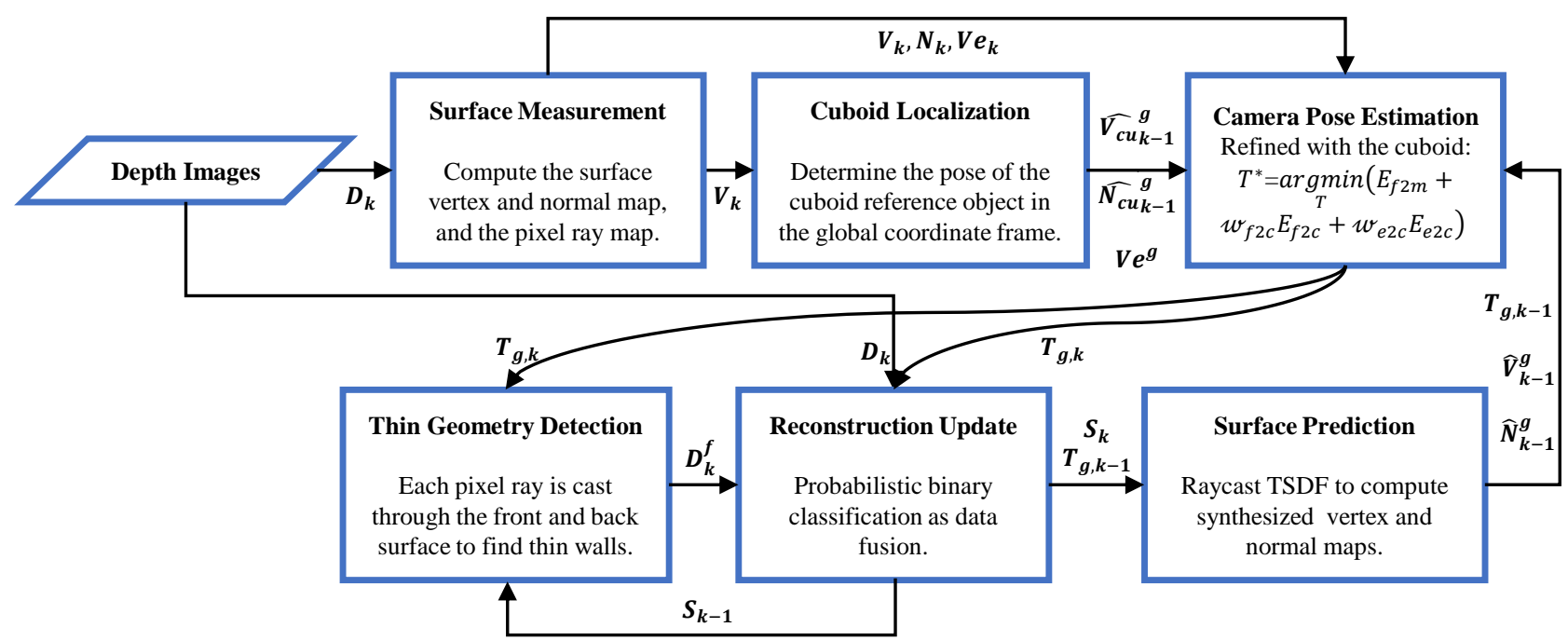

Figure 1. An overview of our system pipeline.

\section{METHOD}

We base our work on the open-sourced implementation of the KinectFusion algorithm from the PCL library [48]. Our reconstruction pipeline is illustrated in Fig. 1, which is described in detail in the following sub-sections.

\section{A. MATHEMATICAL NOTATION}

We define the image domain as $\Omega \subset \mathbb{N}^{2}$, and a depth image at time $k$ is defined as $D_{k}: \Omega \rightarrow \mathbb{R}$, where each single pixel $p \in$ $\Omega$ in the image stores the distance from the camera to the surface. Assuming the camera intrinsic matrix is known, we define the projection and de-homogenization function $\pi$ : $p=\pi(P)$ to map a $3 \mathrm{D}$ point $P=(x, y, z)^{T} \in \mathbb{R}^{3}$ in the camera coordinate onto the image location $p=(u, v)^{T} \in \mathbb{N}^{2}$. We present the 6 degrees-of-freedom (6DOF) camera pose at time $k$ in the global coordinate frame by a rigid transformation matrix:

$$
T_{g, k}=\left[\begin{array}{cc}
R_{g, k} & t_{g, k} \\
0^{T} & 1
\end{array}\right] \in \mathbb{S E}(3)
$$

with a $3 \times 3$ rotation matrix $R_{g, k} \in \mathbb{S} \mathbb{O}(3)$ and a $3 \times 1$ translation vector $t_{g, k} \in \mathbb{R}^{3}$, which transforms a point $P_{k} \in$ $\mathbb{R}^{3}$ in the camera coordinate frame to a global point $P_{g}=$ $R_{g, k} P_{k}+t_{g, k} \in \mathbb{R}^{3}$. For simplicity, we omit the conversion between the 3-vectors and their corresponding homogeneous 4-vectors. A depth pixel p can be back-projected to the global coordinate frame: $P_{g}=T_{g, k} \pi^{-1}\left(p, D_{k}(p)\right)$. An organized vertex map $V_{k}$ is computed by bilateral-filtering and backprojecting the raw depth image $D_{k}$, and its corresponding normal map $N_{k}$ is computed using the Principal Component Analysis (PCA) method.

\section{B. CUBOID LOCALIZATION AS INITIALIZATION}

Given a depth image $D_{k}$ and the reference cuboid with edge lengths $L_{c u}=(a, b, c)$ present in the image, we localize the cuboid and calculate its pose in the global coordinate frame. Live depth frames will be latterly aligned against it when scanning around it to mitigate the accumulating camera drift.

We first perform plane segmentation using the Agglomerative Hierarchical Clustering (AHC) algorithm [49]. Then we check the orthogonality of the segmented planes. Two planes are considered to be orthogonal if the angle $\Theta_{p}$ between their normal vectors is approximately $90^{\circ}$ (i.e. $\left|\Theta_{p}-90^{\circ}\right|<$ $\varepsilon_{\Theta} ; \varepsilon_{\Theta}=5^{\circ}$ ). Once we find three planes that are orthogonal to each other, we check the length of the intersecting line segments between the planes. If the three line segments' lengths match the cuboid edge length parameter $\mathcal{P}_{c u}$ approximately differences below a threshold $\left(\varepsilon_{\mathcal{P}}=10 \mathrm{~mm}\right)$, we claim to find the cuboid and mark the three planes as its adjacent planes.

We consequently define the cuboid coordinate frame of reference. We set frame origin $O_{c u}$ to the intersection point of the three orthogonal planes, and draw the system axes from the normal vectors. Due to the inaccuracy of the depth measurement and camera intrinsic calibration, orthogonality between the normal vectors of the segmented adjacent planes are not guaranteed strictly. We obtain the nearest orthogonal axes $\left[X_{c u}, Y_{c u}, Z_{c u}\right]$ of the frame by solving the Orthogonal Procrustes Problem, where $X_{c u}$ et al. are $3 \times 1$ column vectors. The cuboid pose in the camera frame at time $k$ is:

$$
\begin{aligned}
T_{k, c u} & =\left[\begin{array}{cc}
R_{k, c u} & t_{k, c u} \\
0^{T} & 1
\end{array}\right] \in \mathbb{S E}(3) \\
R_{k, c u} & =\left[X_{c u}, Y_{c u}, Z_{c u}\right] \\
t_{k, c u} & =O_{c u}^{T}
\end{aligned}
$$


Assuming the camera pose $T_{g, k}$ at time $\mathrm{k}$ is known, the cuboid pose $T_{g, c u}$ in the global frame of coordinate could then be derived: $T_{g, c u}=T_{g, k} T_{k, c u}$. Fig. 2 illustrates the notations used in the paper.

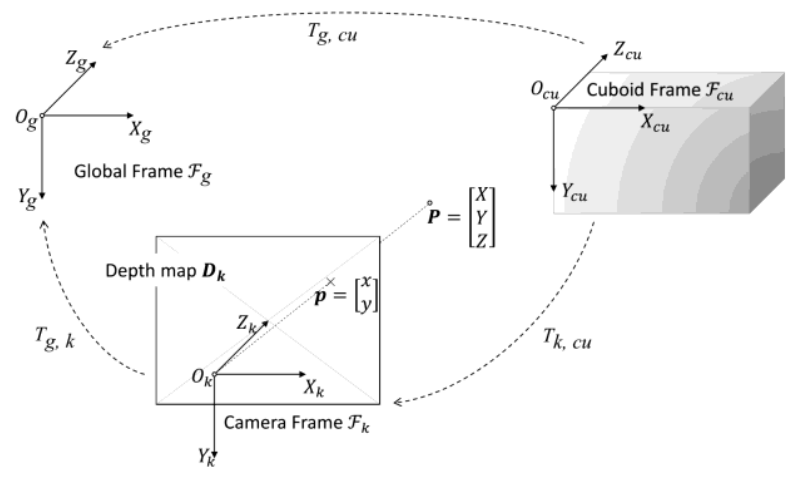

Figure 2. Illustration of the notations used in this paper.

Note that the cuboid pose in the global frame is only evaluated once when we find a triplet of orthogonal planes and left unchanged afterwards. Each incoming depth image is aligned against it to reduce camera drift, as described in the following sections.

\section{CAMERA POSE ESTIMATION}

Since we use depth maps as input sequences, only geometric alignment is performed. For each input frame $D_{k}$ at time $k$, we estimate the current camera pose $T_{g, k}$ by registering the live depth map to both the globally reconstructed surface model and the reference cuboid.

\section{1) FRAME TO MODEL REGISTRATION}

Given the implicit TSDF surface model $S$ and the previously estimated camera pose $T_{g, k-1}$ at time $k-1$, an organized vertex and normal map $\left(\widehat{V}_{k-1}, \widehat{N}_{k-1}\right)$ could be obtained via per-pixel raycast, and then transformed into the global frame as $\left(\widehat{V}_{k-1}^{g}, \widehat{N}_{k-1}^{g}\right)$. For frame-to-model registration, a transformation $\mathrm{T}_{g, k}$ is pursued to minimize the point-to-plane error between $T_{g, k} V_{k}$ and $\widehat{V}_{k-1}^{g}$ :

$$
\begin{aligned}
& E_{f 2 m}\left(T_{g, k}\right)=\sum_{(p, \hat{p}) \in \mathbb{K}_{1}}\left(\left(T_{g, k} V_{k}(p)-\right.\right. \\
& \left.\left.\widehat{V}_{k-1}^{g}(\hat{p})\right) \widehat{N}_{k-1}^{g}(\hat{p})\right)^{2}
\end{aligned}
$$

where $\mathbb{K}_{1}=\{(p, \hat{p})\}$ is the set of correspondences associated by projective data association [1]:

$$
\hat{p}=\pi\left(\widetilde{T}_{k-1, k} V_{k}(p)\right)
$$

where $\widetilde{T}_{k-1, k}$ denotes the transformation from current time $k$ to time $(k-1)$ during each ICP iteration.

\section{2) FRAME TO CUBOID REGISTRATION}

Assuming the cuboid pose has previously been initialized, for each camera pose $T_{g, k-1}$, per-pixel ray casting is performed on the reference cuboid to synthesize a proxy depth map $\widehat{D}_{k-1}^{c u}$. An organized vertex and normal map in the global frame as $\left({\widehat{V_{c u}}}_{k-1}^{g},{\widehat{N_{c u}}}_{k-1}^{g}\right)$ is then derived by back-projecting the proxy depth map and transforming local maps to global space. Similar to the frame-to-model registration, the distance between the current depth measurement and the cuboid surface (frame-to-cuboid) is minimized:

$$
\begin{aligned}
& E_{f 2 c}\left(T_{g, k}\right)=\sum_{(p, \hat{p}) \in \mathbb{K}_{2}}\left(\left(T_{g, k} V_{k}(p)-\right.\right. \\
& \left.\left.{\widehat{V_{c u}}}_{k-1}^{g}(\hat{p})\right){\widehat{N_{c u_{k-1}}}}^{g}(\hat{p})\right)^{2}
\end{aligned}
$$

In addition, we exploit the edge-to-contour distance as a constraint term to mitigate the potential camera drift. Contours of the reference cuboid could be discretized into a 3D point set $V e_{g}^{c u}$ in the global frame with an interval of $1 \mathrm{~mm}$, once the cuboid is successfully localized. Given the inpainted depth map $D_{k}^{\prime}$, we find the edge pixel set $C_{k}$ at depth discontinuities in the live depth map, as proposed in [33]. The 3D edge point set $V e_{k}$ could then be derived by back-projection of $C_{k}$. The edge-to-contour error to minimize is:

$$
\begin{aligned}
& E_{e 2 c}\left(T_{g, k}\right)=\sum_{(s, t) \in \mathbb{K}_{3}}\left(\left(T_{g, k} V e_{k}(s)-\right.\right. \\
& \left.\left.V e_{g}^{c u}(t)\right) \widehat{N}_{c u_{k-1}}^{g}(t)\right)^{2}
\end{aligned}
$$

where $\mathbb{K}_{3}=\{(s, t)\}$ is the correspondence set obtained by nearest neighbor search with KD-tree.

\section{3) JOINT OPTIMIZATION}

We combine (5), (7), and (8) to form a joint cost function:

$$
E_{\text {track }}=E_{f 2 m}+w_{f 2 c} E_{f 2 c}+w_{e 2 c} E_{e 2 c}
$$

where $w_{f 2 c}$ and $w_{e 2 c}$ are the weights that determine the influence of correspondences on the cuboid surfaces and contours. When setting $w_{f 2 c}=w_{e 2 e}=0$, our optimization objective is equivalent to KinectFusion. We set $w_{f 2 c}=1$ and $w_{e 2 e}=4$ in our experiments empirically, enforcing the constraint of the contour correspondences. We compute the camera pose $T_{g, k}$ by minimizing the linear approximation [6] of the overall cost function $\mathrm{E}_{\text {track }}$ iteratively.

\section{POSE GRAPH OPTIMIZATION}

We build pose graphs for optimization based on the geometric characteristics. Each time a triplet of mutually orthogonal planes of the cuboid is observed in the global frame, the orthogonal normal vectors span the space $\mathbb{R}^{3}$, during which strong the geometric constraints ensure accurate camera pose estimation. We select a keyframe as a vertex of the pose graph each time a new trihedron enters the camera's field of view and the relative transformation from the inter-frame alignment as the edges of the graph. We optimize the pose graph using the open-source framework "g2o" [50], and compare our camera trajectories with the optimized ones, showing that our camera poses are accurate enough even without graph optimization. 


\section{E. SURFACE RECONSTRUCTION}

GT In this paper we introduce a method which is an improvement to [18]. We detect the sharp or thin geometric zones by raycasting through the negative TSDF area. Similar to [18], we substitute the simple moving average data fusion strategy with a non-uniform one. We turn the problem of data fusion into a probabilistic binary classification problem, and adopt a denoising scheme, leading to more accurate and cleaner mesh models than other methods.

\section{1) TRUNCATED SIGNED DISTANCE FUNCTION}

The signed distance function (SDF) $F: \mathbb{R}^{3} \rightarrow \mathbb{R}$ introduced in [42] represents the scene in a non-parametric way. Two components are stored at each location of the volume $S$ : the SDF value $F$ and a weight $W$ :

$$
S \mapsto[F, W]
$$

Each SDF value in the voxel corresponds to the signed distance from the cell to the closest surface. In most volumetric reconstruction systems the projective SDF is computed along the view ray, which is view dependent. Instead, we multiply the projective SDF by the cosine value of the incidence angle of each view ray for an approximation of the real SDF value.

The truncated SDF (TSDF) is obtained by normalizing and truncating the SDF value with a constant truncation distance $\delta$. The value of $\delta$ is usually set empirically. When we set it large, the reconstruction is more noise-resistant, whereas the surface details are lost, and when it is set small, the case is just the opposite. Based on the observation that depth measurements near image edges is highly uncertain, we choose an adaptive truncation distance $\delta_{a}$ to ensure fineness of the details of surface reconstruction. Given a 3D point $P$ in the global frame, its TSDF is computed as follows:

$$
\begin{aligned}
& F_{\text {proj }}(P)=D(p)-P_{z} \\
& p=\pi\left(T_{g, k}^{-1} P\right) \\
& \eta=F_{\text {proj }}(P) * \cos \left(\theta_{\text {in }}\right) \\
& F(P)=\left\{\begin{array}{cc}
\min (1, \eta / \delta) & \text { iff } \eta \geq-\delta_{a} \\
\operatorname{null} & \text { otherwise }
\end{array}\right. \\
& \delta_{a}=\max (\sigma, \min (1, \lambda / \Lambda)) * \delta \\
& \lambda=\operatorname{dist}(p) * P_{z} / f
\end{aligned}
$$

where $P_{z}$ is the depth of point $P$ in the camera frame, $\eta$ the approximation of the real SDF value, $\theta_{\text {in }}$ the incidence angle of the view ray, and $f$ the camera focal length. The dist function performs edge detection and distance transformation, to determine the distance from a pixel to its nearest edge. The pixel distance is converted to physical distance $\lambda$ and normalized by a constant physical length $\Lambda$. When $\lambda<\Lambda$, we say the pixel is near the depth edge, and the truncation distance $\delta_{\mathrm{a}}$ is adapted to a smaller value. We empirically set $\sigma=0.3$, $\Lambda=30 \mathrm{~mm}$.

\section{2) RAYCAST FOR THIN GEOMETRIES DETECTION}

Data fusion around the thin geometric zones may be problematic. To detect the thin areas efficiently, we check whether zero crossing is found twice when performing per pixel raycast Different from the raycast procedure in KinectFusion, the ray marching does not stop when $\mathrm{a}+\varepsilon$ to $-\varepsilon$ zero crossing for a visible surface is found. It stops when a $-\varepsilon$ to $+\varepsilon$ back face is found, or when a - ve to a void cell of zero weight, or when finally exists the working volume. Both the latter two cases indicate no thin geometry is met temporarily along the current ray.

Our raycast process outputs a "fake" depth map $D^{f}$ consisting of both positive and negative values. The sign of a value indicates whether a ray finally intersects the front or back surface, and the absolute depth value shows the distance between the camera and the intersection point along the principal optical axis.

Moreover, our modified raycast for thin geometry detection does not march along straight lines all the time. When a pixel ray interfaces the surface at a grazing angle (above a threshold, empirically $60^{\circ}$ ), ray refraction is performed. This is because when a surface is observed at a grazing angle, the ray goes nearly parallel to the surface, resulting in erroneous zero crossing detection. The ray refraction is performed by simply weighted averaging the original pixel ray and the surface normal vector at the incident point. Let $L_{g}(p)$ denote the view ray along pixel $p$, and $N_{g}(p)$ the surface normal at pixel $p$ in the global frame, the refracted view ray $L_{g}^{\prime}(p)$ is updated by:

$$
L_{g}^{\prime}(p)=\left(1-\rho_{r}\right) L_{g}(p)-\rho_{r} N_{g}(p)
$$

where we set $\rho_{r}=1 / 3$ empirically. Fig. 3 illustrates our modified raycast strategy at different incidence angles.

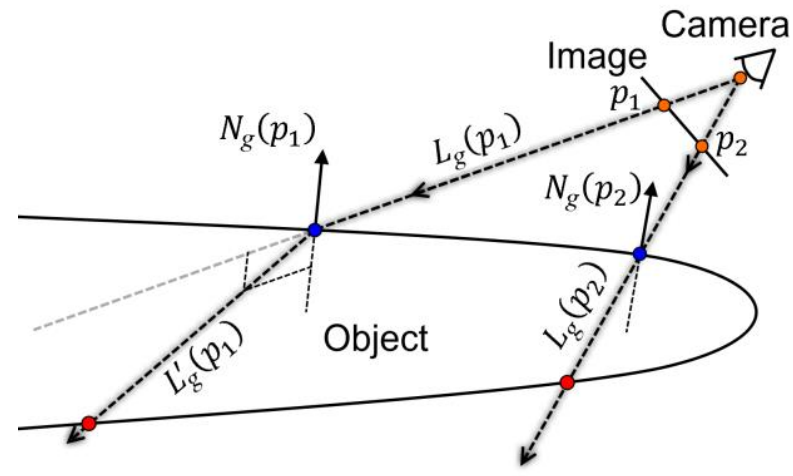

Figure 3. Our modified raycast for thin geometry detection. Note that the ray along pixel $p_{1}$ is refracted closer to the surface normal at the incident point on the surface, due to the large incidence angle, which makes it more quickly to get through the object. The ray along pixel $p_{2}$ marches straight forward without refraction.

\section{3) DATA FUSION AS CLASSIFICATION}

A voxel grid locates around the thin geometric zones may be seen from opposite perspectives, resulting in quite different TSDF measurements. For example in Fig. 4, voxel $P$ is found in the back of and far away from the surface when viewed 
from the left, with a negative TSDF value $F_{1}$ of large magnitude. When the camera orbits to the right side, on the contrary, $P$ is measured in front of the surface with a small positive TSDF value $F_{2}$. Since a TSDF represents the distance from a voxel to its nearest surface, $F_{2}$ is closer to the truthvalue of $P$. However, averaging $F_{1}$ and $F_{2}$ carelessly produces a negative TSDF value, which is not correct. This is the common reason why high-frequency geometries are often smoothed or deformed.

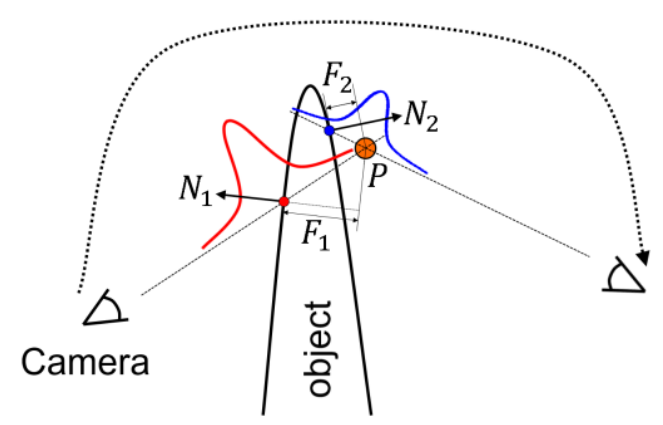

Figure 4. Illustration of the cause of deformation around thin geometries. The voxel $P$ is considered in the back of the surface when firstly viewed from the left, whereas it is found in front of another surface when the camera orbits to the opposite side. Averaging these observations lead to erroneous TSDF results. To address this problem, we propose a probabilistic binary classification strategy for anisotropic data fusion.

Since we have recognized the thin geometries with our raycast procedure in advance, we could address this problem by fusing data around sharp zones efficiently in an anisotropic manner. Apart from the original volume $S$, we maintain a "ghost" volume $S^{\prime}$ as extra storage, so the TSDF of each voxel is determined by both the two volumes. At time $k$, for each voxel $P$ in the global frame, we first transform and project it to an image pixel $p$, then check the value of $D_{k}^{f}(p)$ and compare it with $D_{k}(p)$ to decide in which volume the current measurement should be fused. Algorithm 1 describes this process in detail as follows:

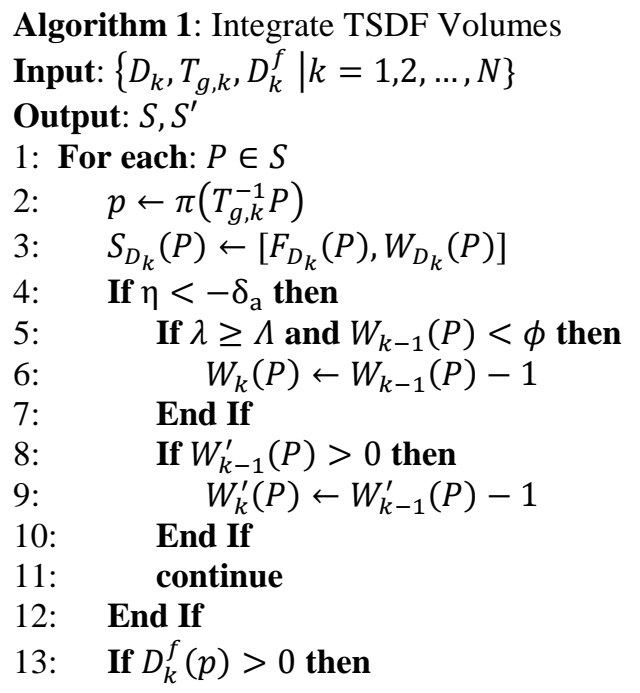

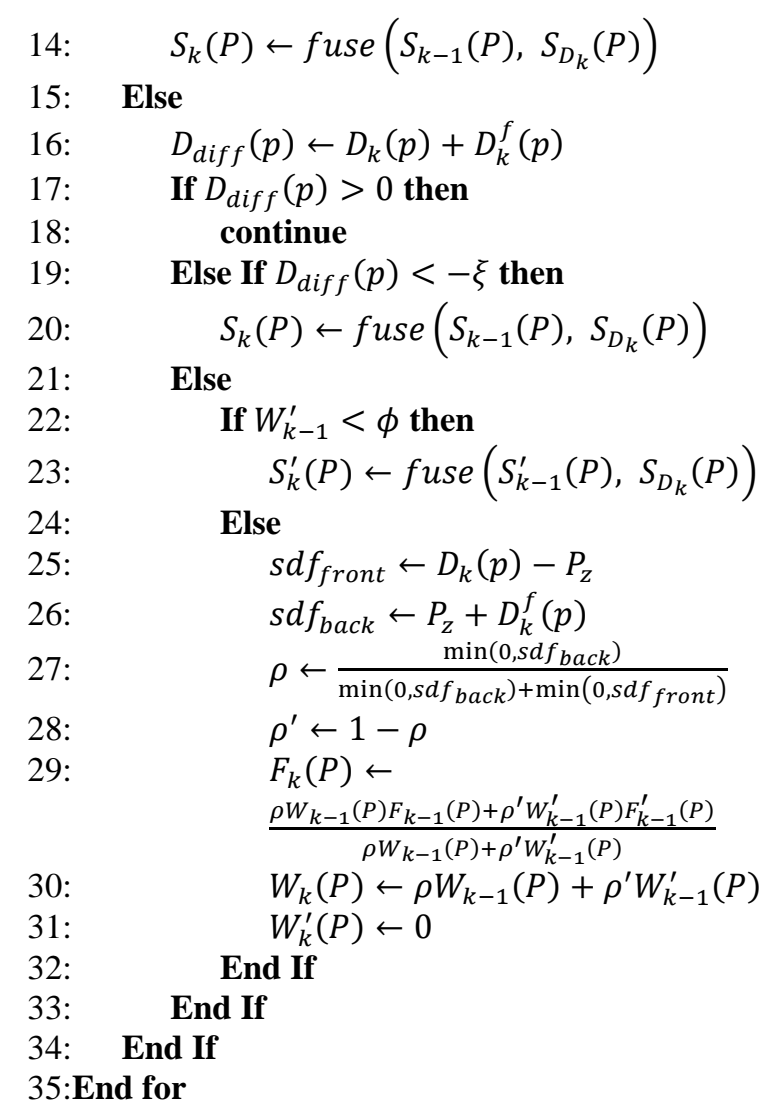

where the fuse function performs a weighted moving average. The threshold $\xi$ denotes the width of a narrow band near the surface, within which the classification strategy is employed. When the difference of the front and back surface $D_{\text {diff }}(p)$ along pixel ray $L_{g}(p)$ lies in the range $[-\xi, 0]$, we try to fuse the incoming data to the "ghost" volume $S^{\prime}$. And when its weight is above a confidence threshold $\phi$, we merge volume $S^{\prime}$ to the main volume $S$, based on the SDF of each voxel $P$ to both the front and back surface. Note that $s d f_{\text {back }}$ is calculated the way opposite to $s d f_{\text {front }}$, resulting in negative $\mathrm{SDF}$ for voxels before (seen from the current camera's viewpoint) the back surface, and positive values behind it.

We finally employ a simple volume denoising scheme for cleaner mesh outputs (Algorithm 1, line 4 12). For each voxel $P$ at time $k$, when $\eta<-\delta_{a}$, we check both the value of $W_{k}$ and $W_{k}^{\prime}$. If $W_{k}$ is below $\phi$ and the corresponding pixel is away from image edges $(\lambda \geq \Lambda)$, or if $W_{k}^{\prime}$ is not zero, we gradually decrease their values by one. This strategy is simple but effective in the presence of highly uncertain depth measurements, e.g. at depth discontinuities or grazing viewing regions.

\section{EVALUATION}

\section{A. DATASET}

In our previous work [18], we have released a dataset CU3D, consists of three synthetic depth image sequences with GT camera trajectories and GT mesh models, and six real-world noisy data sequences with 3D-printed GT models but no GT 
camera trajectories. We extend the dataset with several new depth sequences by scanning some models obtained from daily life, including four dinosaur toys (a Stegosaurus, a Spinosaurus, a Pterosaur, and a Diplodocus. The former three are soft rubber products, but the last one is a rigid body) and a small cardboard box, as shown in Fig. 5. We choose these objects because of their challenging structural details, e.g., the sharp claws, tail tips, and thin spines of the toy dinosaurs, and the thin cardboard walls. Since we have no per-vertex GT for these objects, we measure the distances between manually picked points, or the thickness of thin structures with a Vernier caliper (to $0.02 \mathrm{~mm}$ ) for quantitative evaluation of the compared algorithms. These measurements include the distance from nose to tail tip of the Stegosaurus (abbreviated as "stego-n2t"), the Spinosaurus (abbrev. "spino-n2t"), and the Diplodocus (abbrev. "diplo-n2t"), the distance from the cranial crest tip to tail tip of the Pterosaur (abbrev. "ptero-c2t"), the thickness of the right forelimb of the Diplodocus (abbrev. "diplo-r-forelimb"), and the thickness of the wall of the cardboard box (abbrev. "box-wall"). The measured results as GT are listed in Table I. Note the scale of our measured objectives varies from hundreds of millimeters to only a few millimeters, which challenges the performance of the compared algorithms.

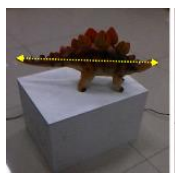

(a)

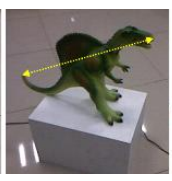

(b)

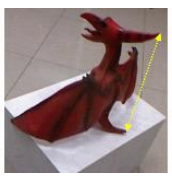

(c)

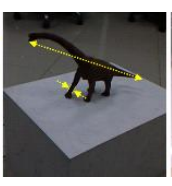

(d)

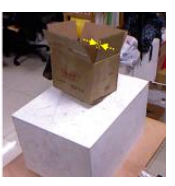

(e)
Figure 5. The new scanned objectives added to our CU3D dataset, including (a) a Stegosaurus, (b) a Spinosaurus, (c) a Pterosaur, (d) a Diplodocus, and (e) a small thin cardboard box. The yellow arrowed dashed lines in each subfigure indicate the manually chosen distance to measure with a Vernier caliper.

TABLE I

Physical Distances Between the Manually Chosen Points MeasURED WITH A VERNIER CALIPER.

\begin{tabular}{ll}
\hline \hline \multicolumn{1}{c}{ Measurement item } & Ground-truth distance $(\mathrm{mm})$ \\
\hline stego-n2t & 491.78 \\
spino-n2t & 589.38 \\
ptero-c2t & 276.80 \\
diplo-n2t & 321.26 \\
diplo-r-forelimb & 12.22 \\
box-wall & 6.24 \\
\hline \hline
\end{tabular}

\section{B. CAMERA TRAJECTORY ACCURACY}

Our reference based odometry algorithm is compared with the following: KinectFusion [1] (PCL's Kinfu implementation [48]), the boundary odometry of Zhou et al. [33], the SDF-2SDF algorithm of Slavcheva et al. [17] (our implementation), and our previous work CuFusion [18]. We compute the absolute trajectory error (ATE) of the 6DOF camera poses on the synthetic depth image sequences - the armadillo, dragon, and bunny sequences from the CU3D dataset. Although planar surfaces of the cuboid occupy the majority of the depth images, all the compared algorithms achieve decent camera trajectories without prominently accumulating drift, as listed in Table II. The ATE of the proposed algorithm in this work is a bit larger (less than $0.5 \mathrm{~mm}$ ) than our previous version of CuFusion, which has little impact on the accuracy of reconstruction. Note that the truncation distance is set to a small value $(5 \mathrm{~mm})$ for most of the cases, whereas when testing Kinfu on sequence "armadillo" it is set up to $25 \mathrm{~mm}$ because small truncation distance in this test case results in severe camera drift and thus failure of the reconstruction. We discuss this phenomenon in the following section $\mathrm{V}$.

TABLE II

COMPARISON OF ATE ON THE SYNTHETIC SEQUENCES OF CU3D.

\begin{tabular}{llll}
\hline \hline \multicolumn{1}{c}{ Algorithm } & Armadillo & Dragon & Bunny \\
\hline KinFu & 3.6 & 3.0 & 4.2 \\
Zhou et al. & 5.6 & 2.9 & 4.3 \\
SDF-2-SDF & 5.7 & 4.4 & 6.6 \\
CuFusion & $\mathbf{1 . 7}$ & $\mathbf{1 . 7}$ & $\mathbf{1 . 3}$ \\
Our approach & $\mathbf{1 . 7}$ & 1.8 & 1.7 \\
\hline \hline
\end{tabular}

\section{COMPARISON WITH POSE GRAPH OPTIMIZATION}

We further compare our online results with those optimized with pose graph optimization. The camera poses where a triplet of mutually orthogonal planes of the reference cuboid is observed, are selected as keyframes and added as fixed vertices into the pose graph. Between which the poses are added as floating vertices and optimized with the "g2o" framework. We test the optimization results on real-world noisy sequences, finding that it has little accuracy gain compared with our online results. Fig. 6 shows the per frame trajectory difference on the sequence "lambunny" of CU3D. The maximum difference is less than $0.1 \mathrm{~mm}$, demonstrating our algorithm outputs comparable camera trajectories to the graph optimized one.

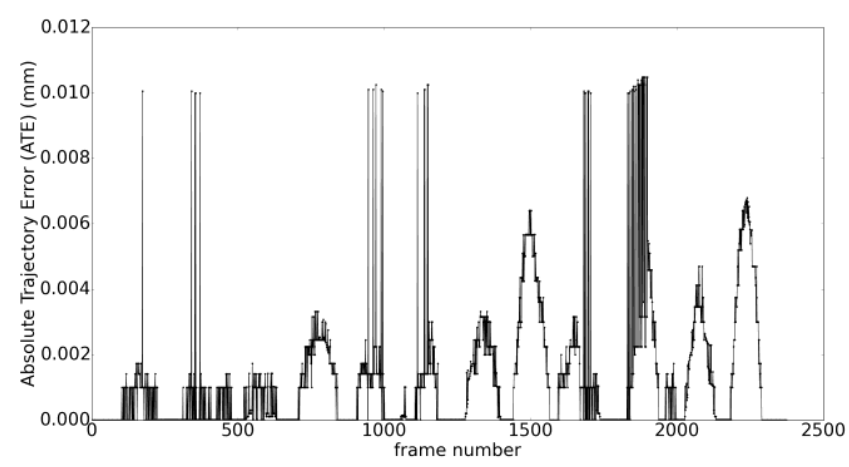

Figure 6. Comparison of per-frame ATE between our algorithm and the graph optimized result on sequence "lambunny". 


\section{SURFACE RECONSTRUCTION ACCURACY}

We quantitatively evaluate the algorithms in the following three ways - the per-vertex error, the physical scale fidelity, and finally the mesh noise of the reconstructions.

\section{1) CLOUD-TO-MESH DISTANCE}

We first test the cloud-to-mesh (C2M) distance from the reconstructtions to the GT models on the three synthetic depth streams and six scanning sequences of the 3D-printed models. Surface reconstructions are first aligned against the GT models, and the $\mathrm{C} 2 \mathrm{M}$ distances in millimeters between the reconstructed and GT models are computed using the CloudCompare software [51]. The C2M distance is quantified by two standard statistics: Mean and Standard deviation (Std.). Fig. 7 plots the reconstructions of the "buddhahead" sequence and their corresponding heat maps of the $\mathrm{C} 2 \mathrm{M}$ distance. Table III provides the error evaluation details of the five compared algorithms on the nine data sequences.

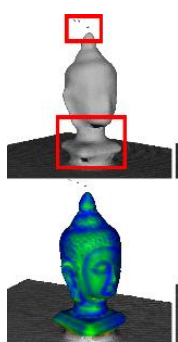

(a)
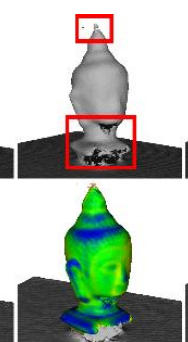

(b)

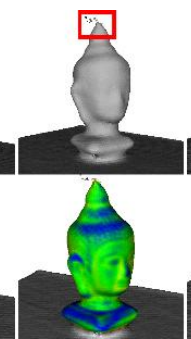

(c)
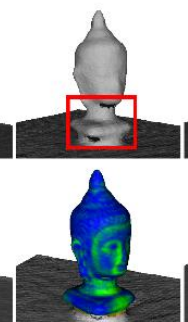

(d)

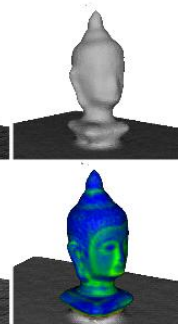

(e)
Figure 7. Qualitative comparison of the algorithms (a) Kinfu, (b) Zhou et al., (c) SDF-2-SDF, (d) CuFusion, and (e) our proposed method on sequence "buddhahead". Top row: the reconstructed mesh models, bottom row: the heat maps corresponding to the $\mathrm{C} 2 \mathrm{M}$ errors.

\section{TABLE III}

SURFACE RECONSTRUCTION ACCURACY ON OUR SYNTHETIC AND REALWORLD DATA, WITH C2M ERROR METRIC (MEAN \pm STD.) IN MILLIMETERS.

\begin{tabular}{lccccc}
\hline \hline $\begin{array}{c}\text { Se- } \\
\text { quence }\end{array}$ & KinFu & $\begin{array}{c}\text { Zhou } \text { et } \\
\text { al. }\end{array}$ & $\begin{array}{c}\text { SDF-2- } \\
\text { SDF }\end{array}$ & $\begin{array}{c}\text { CuFu- } \\
\text { sion }\end{array}$ & $\begin{array}{c}\text { Our ap- } \\
\text { proach }\end{array}$ \\
\hline $\begin{array}{c}\text { arma- } \\
\text { dillo }\end{array}$ & $1.0 \pm 1.1$ & $0.2 \pm 0.2$ & $0.4 \pm 0.3$ & $\mathbf{0 . 2} \pm \mathbf{0 . 1}$ & $0.2 \pm 0.2$ \\
dragon & $0.2 \pm 0.2$ & $0.1 \pm 0.1$ & $0.4 \pm 0.3$ & $0.1 \pm 0.1$ & $\mathbf{0 . 1} \pm \mathbf{0 . 1}$ \\
bunny & $0.2 \pm 0.4$ & $0.2 \pm 0.4$ & $0.4 \pm 0.4$ & $\mathbf{0 . 1} \pm \mathbf{0 . 1}$ & $0.2 \pm 0.3$ \\
mug & $1.4 \pm 1.1$ & $1.4 \pm 0.9$ & $0.8 \pm 0.9$ & $0.9 \pm 0.8$ & $\mathbf{0 . 7} \pm \mathbf{0 . 8}$ \\
lam- & $\mathbf{0 . 9} \pm \mathbf{1 . 2}$ & $1.1 \pm 1.2$ & $1.4 \pm 1.1$ & $1.3 \pm 1.2$ & $1.1 \pm 1.1$ \\
bunny & $2.4 \pm 1.1$ & $1.0 \pm 1.0$ & $2.2 \pm 1.2$ & $1.2 \pm 1.1$ & $\mathbf{0 . 9} \pm \mathbf{1 . 2}$ \\
owl \\
tooth & $2.7 \pm 1.4$ & $1.3 \pm 1.3$ & $1.3 \pm 1.3$ & $1.5 \pm 1.5$ & $\mathbf{1 . 3} \pm \mathbf{1 . 3}$ \\
wingedc & $1.5 \pm 2.0$ & $1.5 \pm 2.0$ & $2.7 \pm 2.6$ & $1.4 \pm 2.2$ & $\mathbf{1 . 3} \pm \mathbf{1 . 9}$ \\
at & & & & \\
buddahe & $1.6 \pm 2.1$ & $3.0 \pm 2.0$ & $2.7 \pm 2.0$ & $\mathbf{1 . 3} \pm \mathbf{2 . 0}$ & $1.5 \pm 2.1$ \\
ad & & & & & \\
\hline \hline
\end{tabular}

\section{2) PHYSICAL SCALE OF THE RECONSTRUCTION}

As can be seen from Table III, on the reconstruction of smallsized objects, it is hard to tell which method has a significant advantage over the others as long as no noticeable camera drift happens. To test the reconstruction fidelity, we also measure the physical scale of the reconstructions (total length or body part thickness) of the five newly added scanning sequences. Distance in millimeters is measured using the point-picking tool of the CloudCompare software. We compare the measuring results with the corresponding GT values listed in Table I, and use the absolute distance (listed in the parentheses) between the measurements and the GT values as an indicator of the accuracy of the tested algorithms, as illustrated in Table IV. Fig. 8 qualitatively demonstrates the reconstruction of the "Diplodocus" and "cardboard box" sequence. Note that the SDF-2-SDF method fails to create the Diplodocus model due to drift in camera trajectory estimation, and neither the Kinfu and Zhou et al.'s method reconstructs the Diplodocus's right forelimb successfully.

TABLE IV

MEASUREMENT RESULTS OF THE MANUALLY CHOSEN OBJECTS' PARTS IN MILLIMETERS. THE ABSOLUTE DISTANCE BETWEEN THE MEASUREMENTS OF MESH MODELS AND THE REAL OBJECTS ARE SHOWN IN THE PARENTHESES BEHIND (SMALLER IS BETTER).

\begin{tabular}{|c|c|c|c|c|c|c|}
\hline Measurement item & $\mathrm{GT}(\mathrm{mm})$ & $\mathrm{KinFu}$ & Zhou et al. & SDF-2-SDF & CuFusion & Our approach \\
\hline stego-n2t & 491.78 & $479.07(12.71)$ & $479.18(12.6)$ & $485.62(6.16)$ & $475.6(16.18)$ & $483.4(8.38)$ \\
\hline spino- $2 \mathrm{t}$ & 589.38 & $595.48(6.1)$ & $601.41(12.03)$ & $595.66(6.28)$ & $596.76(7.38)$ & $592.98(3.60)$ \\
\hline diplo-n2t & 321.26 & $293.91(27.35)$ & $306.66(14.6)$ & -- - - & $300.29(20.97)$ & $316.63(4.63)$ \\
\hline diplo-r-forelimb & 12.22 & -- - 2 - & -- & -- & $6.7(5.52)$ & $12.1(0.12)$ \\
\hline box-wall & 6.24 & $6.66(0.42)$ & $7.32(1.08)$ & $5.92(0.32)$ & $11.63(5.39)$ & $6.76(0.52)$ \\
\hline
\end{tabular}




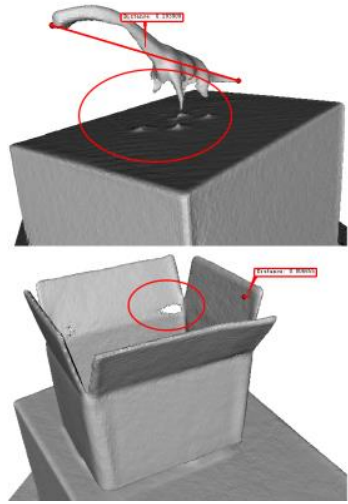

(a)

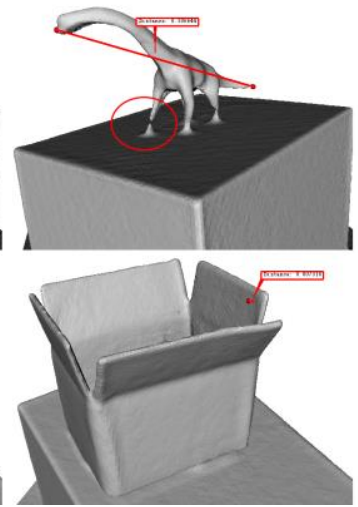

(b)

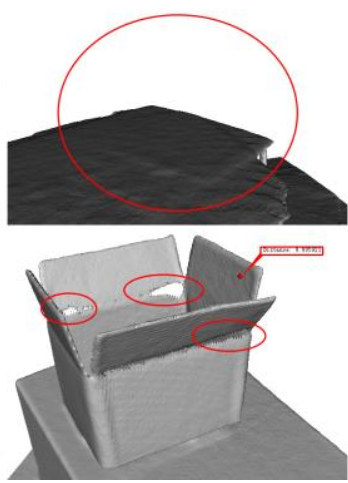

(c)

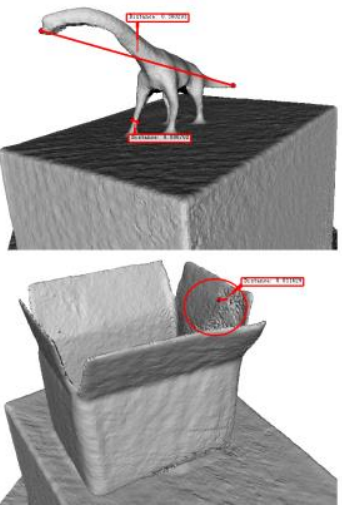

(d)

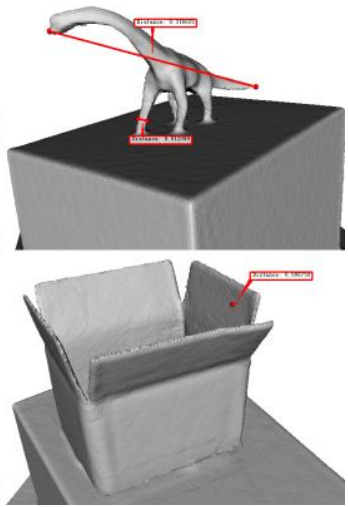

(e)

Figure 8. Qualitative comparison of the algorithms (a) Kinfu, (b) Zhou et al., (c) SDF-2-SDF, (d) CuFusion, and (e) our proposed method on sequence "Diplodocus" (top row) and sequence "cardboard box" (bottom row).

\section{3) MESH NOISE}

We finally compare the mesh and point cloud generated by the evaluated algorithms. For applications such as 3D printing, clean and topologically consistent mesh output would be of vital importance. Thanks to the accurate camera pose estimation and the denoising strategy during the data fusion process, our method is able to generate globally consistent mesh models superior to others. Fig. 9 demonstrates the reconstruction of the compared methods on sequence "Spinosaurus", where sectional views are provided for visualizing both sides of the surface. As can be seen from the sectional views, our mesh model contains the least noise and outliers among the compared algorithms.

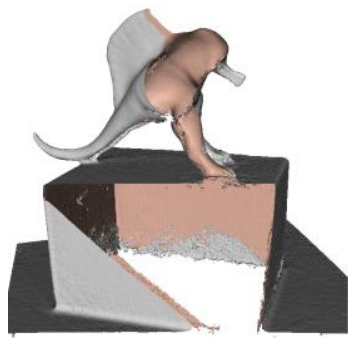

(a)

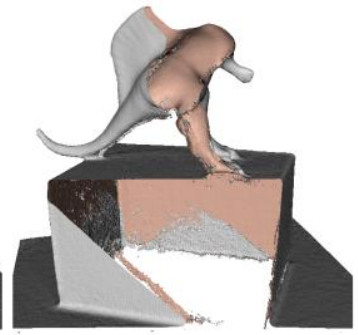

(b)

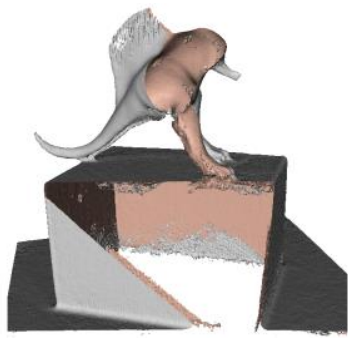

(c)

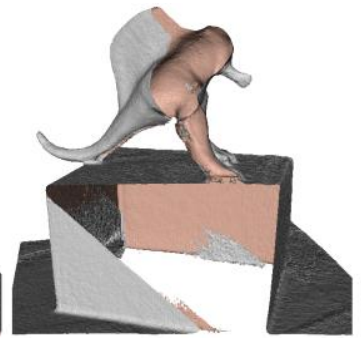

(d)

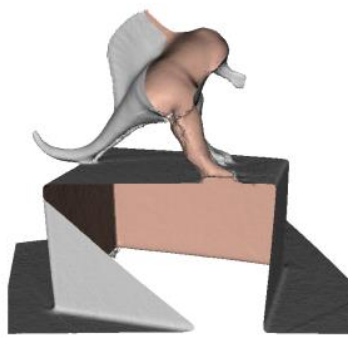

(e)

Figure 9. Sectional views of the algorithms (a) Kinfu, (b) Zhou et al., (c) SDF-2-SDF, (d) CuFusion, and (e) our proposed method on reconstruction of sequence "Spinosaurus".

TABLE V

FACE AND VERTEX (LISTED IN THE PARENTHESES) COUNT OF THE SURFACE MODELS GENERATED BY THE COMPARED ALGORITHMS. SMALLER VALUE IS BETTER IF NO DRASTIC FAILURE OF THE RECONSTRUCTION HAPPENS, INDICATING CLEANER MODEL OUTPUT AS DEMONSTRATED IN FIG. 9.

\begin{tabular}{|c|c|c|c|c|c|}
\hline Sequence & $\mathrm{KinFu}$ & Zhou et al. & SDF-2-SDF & CuFusion & Our approach \\
\hline armadillo & 387601 (194909) & $380642(191541)$ & 382897 (192600) & 382161 (192372) & 380234 (191255) \\
\hline dragon & $427022(216153)$ & 425901 (215487) & $432404(217893)$ & $421269(213688)$ & $422416(213238)$ \\
\hline bunny & 381912 (192908) & 382453 (193282) & 385194 (193916) & 381273 (192264) & 381602 (192551) \\
\hline mug & 371373 (194913) & $395920(213064)$ & 378467 (198830) & 349920 (181024) & $328169(166157)$ \\
\hline lambunny & 390045 (210086) & $399374(215993)$ & 374001 (196184) & 347144 (179696) & $324172(165600)$ \\
\hline owl & $373064(200120)$ & $381390(204482)$ & 362957 (186951) & 364773 (188779) & $333662(171376)$ \\
\hline tooth & $374547(202142)$ & 399135 (216662) & 368753 (192225) & 365439 (188833) & $327058(166900)$ \\
\hline wingedcat & 404647 (220129) & $404900(220328)$ & $497058(257532)$ & 371897 (193442) & 336911 (171508) \\
\hline buddhahead & $411831(222773)$ & $445290(241586)$ & 417893 (219216) & $389424(200526)$ & 364055 (184930) \\
\hline Stegosaurus & $495461(264761)$ & $482179(259652)$ & $827302(421941)$ & $467333(240470)$ & $400986(204506)$ \\
\hline Spinosaurus & $395476(209303)$ & $417042(221981)$ & 389946 (198736) & $391549(201743)$ & $345417(175003)$ \\
\hline Pterosaur & $462870(245659)$ & $477072(254872)$ & 623249 (321049) & $445836(227873)$ & 415591 (209501) \\
\hline Diplodocus & $517564(274194)$ & $421678(223022)$ & -- & 407979 (211236) & $364548(184535)$ \\
\hline Cardboard box & $515780(273150)$ & $532378(282620)$ & 600319 (320499) & 501039 (259089) & $449509(228410)$ \\
\hline
\end{tabular}


Table V illustrates the number of triangle faces and vertices (listed in the parentheses) of each model generated by the algorithms for each depth stream of our CU3D dataset (currently 14 sequences in total). On the first three synthetic sequences, the statistical differences are around $1 \%$ since there is no noise in the input depth images. On the real-world sequences however, the differences reach up to $10 \%$ 30\%. Our algorithm generates the least outliers in the point clouds as well as the triangle meshes on all of the noisy sequences, proving the effectiveness of our denoising scheme.

\section{DISCUSSION AND CONCLUSION}

In this paper we propose an approach for accurate and clean object reconstruction. Given a stream of depth images and a known cuboid reference object present in the scene, we keep drift free camera pose estimation with the constraints added by the reference object, without the need of pose graph optimization. We then fuse the living data into one globally consistent model in real-time by transforming the problem of data fusion into a probabilistic binary classification problem, and keep the reconstruction fidelity in particular highly curved and concave zones of the scanned objectives, preserving the surface smoothness and cleanliness utilizing a simple denoising strategy - especially in areas invisible near the surface. Our method takes one step further for the applications which demand fine-grained reconstruction details such 3D printing.

One limitation of our method is that it assumes the scene or object is static during scanning. Any deformation or motion of the objects may lead to failure of the reconstruction, especially in the presence of thin geometries. Currently the reconstruction of sharp zones in motion remains a challenge to us. About the memory consumption, our method costs 8 bytes per voxel - half the memory cost of our previous work, CuFusion, still twice as much as KinectFusion at the same resolution. Our future work will focus on the memory efficiency of the volumetric representation, enabling higher volume resolution and a larger scale of reconstruction. Also, we will explore the fidelity preservation of thin geometry in motion, to make the system work with challenging scenes. 


\section{REFERENCES}

[1] R. A. Newcombe et al., "KinectFusion: Real-time Dense Surface Mapping and Tracking," in Proceedings of the 2011 10th IEEE International Symposium on Mixed and Augmented Reality, Washington, DC, USA, 2011, pp. 127-136.

[2] P. J. Besl and N. D. McKay, "A method for registration of 3-D shapes," IEEE Trans. Pattern Anal. Mach. Intell., vol. 14, no. 2, pp. 239-256, Feb. 1992.

[3] Y. Chen and G. Medioni, "Object Modelling by Registration of Multiple Range Images," Image Vis. Comput, vol. 10, no. 3, pp. 145155, Apr. 1992.

[4] W. E. Lorensen and H. E. Cline, "Marching Cubes: A High Resolution 3D Surface Construction Algorithm," SIGGRAPH Comput Graph, vol. 21, no. 4, pp. 163-169, Aug. 1987.

[5] S. Meister, S. Izadi, P. Kohli, M. Hämmerle, C. Rother, and D. Kondermann, "When can we use kinectfusion for ground truth acquisition," in Proc. Workshop on Color-Depth Camera Fusion in Robotics, 2012, vol. 2.

[6] K.-L. Low, "Linear least-squares optimization for point-to-plane icp surface registration," Chap. Hill Univ. N. C., vol. 4, 2004.

[7] A. Segal, D. Haehnel, and S. Thrun, "Generalized-ICP.," in Robotics: science and systems, 2009, vol. 2, p. 435.

[8] T. Whelan, M. Kaess, M. Fallon, H. Johannsson, J. Leonard, and J. McDonald, "Kintinuous: Spatially extended kinectfusion," 2012.

[9] T. Weise, T. Wismer, B. Leibe, and L. Van Gool, "In-hand scanning with online loop closure," in Computer Vision Workshops (ICCV Workshops), 2009 IEEE 12th International Conference on, 2009, pp. 1630-1637.

[10] T. Whelan, S. Leutenegger, R. S. Moreno, B. Glocker, and A. Davison, "ElasticFusion: Dense SLAM Without A Pose Graph," in Proceedings of Robotics: Science and Systems, Rome, Italy, 2015.

[11] T. Whelan, M. Kaess, H. Johannsson, M. Fallon, J. J. Leonard, and J. McDonald, "Real-time large-scale dense RGB-D SLAM with volumetric fusion," Int. J. Robot. Res., vol. 34, no. 4-5, pp. 598-626, 2015.

[12] O. Kähler, V. A. Prisacariu, and D. W. Murray, "Real-time largescale dense 3d reconstruction with loop closure," in European Conference on Computer Vision, 2016, pp. 500-516.

[13] S. Choi, Q.-Y. Zhou, and V. Koltun, "Robust reconstruction of indoor scenes," in Proceedings of the IEEE Conference on Computer Vision and Pattern Recognition, 2015, pp. 5556-5565.

[14] Q.-Y. Zhou and V. Koltun, "Dense scene reconstruction with points of interest," ACM Trans. Graph. ToG, vol. 32, no. 4, p. 112, 2013.

[15] T. Whelan, R. F. Salas-Moreno, B. Glocker, A. J. Davison, and S. Leutenegger, "ElasticFusion: Real-time dense SLAM and light source estimation," Int. J. Robot. Res., vol. 35, no. 14, pp. $1697-$ 1716,2016

[16] M. Slavcheva, W. Kehl, N. Navab, and S. Ilic, "SDF-2-SDF: highly accurate 3D object reconstruction," in European Conference on Computer Vision, 2016, pp. 680-696.

[17] M. Slavcheva, W. Kehl, N. Navab, and S. Ilic, "SDF-2-SDF Registration for Real-Time 3D Reconstruction from RGB-D Data," Int. J. Comput. Vis., pp. 1-22, 2018.

[18] C. Zhang and Y. Hu, "CuFusion: Accurate real-time camera tracking and volumetric scene reconstruction with a cuboid," Sensors, vol. 17, no. 10, p. 2260, 2017.

[19] A. J. Davison, "Real-time simultaneous localisation and mapping with a single camera," in null, 2003, p. 1403.

[20] G. Klein and D. Murray, "Parallel tracking and mapping for small AR workspaces," in Mixed and Augmented Reality, 2007. ISMAR 2007. 6th IEEE and ACM International Symposium on, 2007, pp. 225-234.

[21] R. A. Newcombe and A. J. Davison, "Live dense reconstruction with a single moving camera," in Computer Vision and Pattern Recognition (CVPR), 2010 IEEE Conference on, 2010, pp. 1498-1505.

[22] J. Stühmer, S. Gumhold, and D. Cremers, "Real-time dense geometry from a handheld camera," in Joint Pattern Recognition Symposium, 2010, pp. 11-20.

[23] R. A. Newcombe, S. J. Lovegrove, and A. J. Davison, "DTAM: Dense tracking and mapping in real-time," in Computer Vision (ICCV), 2011 IEEE International Conference on, 2011, pp. 23202327.
[24] S. Izadi et al., "KinectFusion: Real-time 3D Reconstruction and Interaction Using a Moving Depth Camera," in Proceedings of the 24th Annual ACM Symposium on User Interface Software and Technology, New York, NY, USA, 2011, pp. 559-568.

[25] J. Sturm, E. Bylow, F. Kahl, and D. Cremers, "CopyMe3D: Scanning and Printing Persons in 3D," in Pattern Recognition - 35th German Conference, GCPR 2013, Saarbrücken, Germany, September 36, 2013. Proceedings, 2013, pp. 405-414.

[26] M. Nießner, M. Zollhöfer, S. Izadi, and M. Stamminger, "Real-time 3D Reconstruction at Scale Using Voxel Hashing," ACM Trans Graph, vol. 32, no. 6, pp. 169:1-169:11, Nov. 2013.

[27] V. A. Prisacariu et al., "InfiniTAM v3: A Framework for LargeScale 3D Reconstruction with Loop Closure," ArXiv Prepr. ArXiv170800783, 2017.

[28] A. Dai, M. Niels sner, M. Zollhöfer, S. Izadi, and C. Theobalt, "Bundlefusion: Real-time globally consistent $3 \mathrm{~d}$ reconstruction using onthe-fly surface reintegration," ACM Trans. Graph. TOG, vol. 36, no. 4, p. 76a, 2017.

[29] H. Roth and M. Vona, "Moving Volume KinectFusion.," in BMVC, 2012, vol. 20, pp. 1-11.

[30] T. Whelan, M. Kaess, J. J. Leonard, and J. McDonald, "Deformation-based loop closure for large scale dense RGB-D SLAM," in Intelligent Robots and Systems (IROS), 2013 IEEE/RSJ International Conference on, 2013, pp. 548-555.

[31] D. Lefloch, M. Kluge, H. Sarbolandi, T. Weyrich, and A. Kolb, "Comprehensive Use of Curvature For Robust And Accurate Online Surface Reconstruction," IEEE Trans. Pattern Anal. Mach. Intell. PAMI, p. 10.1109/TPAMI.2017.2648803, 2017.

[32] W. Dong, Q. Wang, X. Wang, and H. Zha, "PSDF Fusion: Probabilistic Signed Distance Function for On-the-fly 3D Data Fusion and Scene Reconstruction," ArXiv Prepr. ArXiv180711034, 2018.

[33] Q.-Y. Zhou and V. Koltun, "Depth camera tracking with contour cues," in 2015 IEEE Conference on Computer Vision and Pattern Recognition (CVPR), 2015, pp. 632-638.

[34] E. Ataer-Cansizoglu, Y. Taguchi, S. Ramalingam, and T. Garaas, "Tracking an RGB-D camera using points and planes," in Proceedings of the IEEE International Conference on Computer Vision Workshops, 2013, pp. 51-58.

[35] Y. Taguchi, Y.-D. Jian, S. Ramalingam, and C. Feng, "Point-plane SLAM for hand-held 3D sensors," in Robotics and Automation (ICRA), 2013 IEEE International Conference on, 2013, pp. 51825189.

[36] M. Kaess, "Simultaneous localization and mapping with infinite planes.," in ICRA, 2015, vol. 1, p. 2.

[37] R. F. Salas-Moreno, B. Glocken, P. H. Kelly, and A. J. Davison, "Dense planar SLAM," in Mixed and Augmented Reality (ISMAR), 2014 IEEE International Symposium on, 2014, pp. 157-164.

[38] L. Ma, C. Kerl, J. Stückler, and D. Cremers, "Cpa-slam: Consistent plane-model alignment for direct rgb-d slam," in Robotics and Automation (ICRA), 2016 IEEE International Conference on, 2016, pp. 1285-1291.

[39] R. F. Salas-Moreno, R. A. Newcombe, H. Strasdat, P. H. Kelly, and A. J. Davison, "Slam++: Simultaneous localisation and mapping at the level of objects," in Proceedings of the IEEE conference on computer vision and pattern recognition, 2013, pp. 1352-1359.

[40] N. Fioraio and L. Di Stefano, "Joint detection, tracking and mapping by semantic bundle adjustment," in Proceedings of the IEEE Conference on Computer Vision and Pattern Recognition, 2013, pp. 15381545 .

[41] A. Elfes and L. Matthies, "Sensor integration for robot navigation: Combining sonar and stereo range data in a grid-based representataion," in 26th IEEE Conference on Decision and Control, 1987, vol. 26, pp. 1802-1807.

[42] B. Curless and M. Levoy, "A Volumetric Method for Building Complex Models from Range Images," in Proceedings of the 23rd Annual Conference on Computer Graphics and Interactive Techniques, New York, NY, USA, 1996, pp. 303-312.

[43] M. Keller, D. Lefloch, M. Lambers, S. Izadi, T. Weyrich, and A. Kolb, "Real-time $3 \mathrm{~d}$ reconstruction in dynamic scenes using pointbased fusion," in 3DTV-Conference, 2013 International Conference on, 2013, pp. 1-8. 
[44] J. Serafin and G. Grisetti, "NICP: Dense normal based point cloud registration," in Intelligent Robots and Systems (IROS), 2015 IEEE/RSJ International Conference on, 2015, pp. 742-749.

[45] H. Pfister, M. Zwicker, J. van Baar, and M. Gross, "Surfels: Surface Elements As Rendering Primitives," in Proceedings of the 27th Annual Conference on Computer Graphics and Interactive Techniques, New York, NY, USA, 2000, pp. 335-342.

[46] J. Sturm, N. Engelhard, F. Endres, W. Burgard, and D. Cremers, "A benchmark for the evaluation of RGB-D SLAM systems," in Intelligent Robots and Systems (IROS), 2012 IEEE/RSJ International Conference on, 2012, pp. 573-580.

[47] A. Handa, T. Whelan, J. B. McDonald, and A. J. Davison, “A Benchmark for RGB-D Visual Odometry, 3D Reconstruction and SLAM," in IEEE Intl. Conf. on Robotics and Automation, ICRA, Hong Kong, China, 2014.

[48] R. B. Rusu and S. Cousins, "3D is here: Point Cloud Library (PCL)," in 2011 IEEE International Conference on Robotics and Automation, 2011, pp. 1-4.

[49] C. Feng, Y. Taguchi, and V. R. Kamat, "Fast plane extraction in organized point clouds using agglomerative hierarchical clustering," in 2014 IEEE International Conference on Robotics and Automation (ICRA), 2014, pp. 6218-6225.

[50] R. Kümmerle, G. Grisetti, H. Strasdat, K. Konolige, and W. Burgard, "g 2 o: A general framework for graph optimization," in Robotics and Automation (ICRA), 2011 IEEE International Conference on, 2011, pp. 3607-3613.

[51] "CloudCompare - Open Source project." [Online]. Available: http://www.danielgm.net/cc/. [Accessed: 19-Jul-2017].

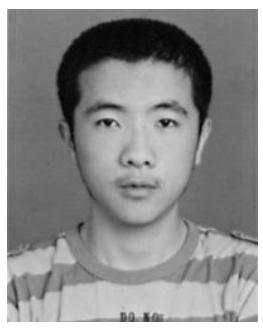

CHEN ZHANG received the B.S. degree in Computer Science from Zhejiang University, China, in 2013. From September 2013, he is studying as a $\mathrm{Ph} . \mathrm{D}$. student in College of Computer Science and Technology, Zhejiang University, China. He is currently working with the Computer Animation \& Perception Group under the State Key Lab of CAD \& CG, Zhejiang University. His primary research interests include simultaneous localization and mapping (SLAM) and 3D reconstruction. 